\title{
Bone Pain
}

National Cancer Institute

\section{Source}

National Cancer Institute. Bone Pain. NCI Thesaurus. Code C38644.

Painful sensation in the bones. 